\title{
Development of automated top-down construction system for low-rise building structures
}

\author{
Aparna HARICHANDRAN ${ }^{1,2}$, Benny RAPHAEL ${ }^{3}$, and Abhijit MUKHERJEE ${ }^{4}$ \\ ${ }_{1}$ PhD Candidate, Department of Civil Engineering, Indian Institute of Technology Madras, Chennai, India \\ ${ }^{2}$ PhD Candidate, School of Civil and Mechanical Engineering, Curtin University, Bently, WA 6102, Australia \\ ${ }^{3}$ Professor, Department of Civil Engineering, Indian Institute of Technology Madras, Chennai, India \\ ${ }^{4}$ Professor, School of Civil and Mechanical Engineering, Curtin University, Bently, WA 6102, Australia
}

Submitted January 2020

Revised May 2020

Accepted May 2020

Published June 2020

\section{Corresponding Author}

Aparna Harichandran

aparnaharichandran@gmail.com

Building Technology and

Construction Management

Division,

Department of Civil Engineering,

Indian Institute of Technology

Madras,

Sardar Patel Road, Chennai,

Tamil Nadu - 600036, India

DOI doi.org/10.29173/ijic217

Pages 22-33

ISSN 2563-5034

Distributed under Creative

Commons CC-BY-NC-ND 4.0

\section{ABSTRACT}

Automation is the best solution for achieving high productivity and quality in the construction industry at reduced cost and time. The main objective of this study is to develop an economical automated construction system (ACS) for low-rise buildings. The incremental development of the construction system and the structural system through different versions of laboratory prototypes are described in this paper. These ACS prototypes adopt a top-down construction method. This method involves the building of the structural system step by step from the top floor to the bottom floor by connecting and lifting structural modules. ACS prototype 1 consist of wooden structural modules and electric motor system. ACS prototype 2 has a highly automated custom designed hydraulic motor system to construct steel structural frame. ACS prototype 3 is a partially automated system where the steel structural modules are connected manually. These prototypes were evaluated on the basis of function, cost and efficiency of operations. Based on overall performance, ACS prototype 3 is identified as the best economical option for the construction of low-rise buildings. When the speed of construction is more important than cost, the ACS prototype 2 is the apt solution. This paper describes the challenges in developing an ACS and the criteria to evaluate its performance. It also includes a preliminary framework for the development of an automated construction monitoring system and its experimental evaluation. This machine learning-based framework is to identify the operations of ACS from sensor measurements using Support Vector Machines. Most of the operations are identified reasonably well and the best identification accuracy is $96 \%$. The future studies are focusing on to improve the accuracy of operation identification, further development of the monitoring system and the ACS for actual implementation in construction sites. 


\section{Introduction and background}

The resistance of the construction industry towards technology adoption is one of the main reasons for its low productivity compared to other industries [1]. Besides, construction sites have been recognized as one of the most unsafe working environments [2]. Improved productivity, enhanced quality, and reduced cost and time of construction are the prime most impetus for automation in construction.

The concept of automating construction dates back to the 1970s. Nonetheless, robotics and automation techniques are not still widely implemented in real construction sites, especially for the construction of low-rise buildings. Rehabilitation of a massive population in situations such as natural calamities demands faster construction of a large number of low-rise buildings. Hence, the development of automation technologies for low-rise building construction demands focused attention.

Some of the main reasons for the low application of automation in construction involve high cost of implementation, lack of skilled labour and low investment in research and development [3]. In addition to the complexity of the interdisciplinary nature of work in construction automation, the knowledge gap between the construction industry and academic research contributes to the low adoption rate of automation in construction.

The research in construction automation is extremely technology-oriented and hence highly expensive. The design of an automation system has to go through several iterative changes depending on the practical issues related to construction. This requires developing simple prototypes and testing them in the laboratory. After all the practical issues are resolved, the concept can be gradually introduced in the field [3].

This paper presents various stages of development of an automated construction system (ACS) intended for the construction of the structural frame of low-rise buildings. This construction system follows the automated top-down construction method [4-6]. The evolution of the construction system and the structural system through different versions of laboratory prototypes are described in this paper. This study intends to account for various constructability issues which are often overlooked in academic research. However, the final implementation of the ACS in actual construction sites might require further refinement. This paper attempts to showcase the challenges in developing an ACS and propose some criteria to evaluate its performance.

The prototypes of the ACS developed during these studies revealed various unsafe scenarios which may lead to severe accidents. This situation compels close monitoring of automated construction operations. A preliminary framework for the development of an automated construction monitoring system is also described in this paper. The proposed framework is to identify ACS operations from sensor measurements. This study is the initial step towards the development of an integrated automated construction monitoring system.

The organization of the paper is given as follows: The second section reviews ACSs implemented in the construction industry, the techniques adopted to monitor them and their limitations. The third section categories and describes the method of automated construction followed in this study. The following section covers various aspects of the incremental development of the structural system and construction system. The preliminary framework for identification of ACS operations is introduced in the fifth section. The following section for results and discussion includes performance evaluation of all ACS prototypes and the proposed identification framework. The final section concludes all the outcomes of the study and involves a brief description of future work.

\section{Existing automated construction systems and monitoring techniques}

Automated construction systems (ACS), even though limited in application, are primarily applied for the construction of high-rise buildings. The central operation unit in automated construction is called 'factory' [7]. It can be located either on the ground level (ground factory) [8] or the top of the building (sky factory) [9] or completely independent of the building under construction. The main factory consists of many subsystems which focus on specific operations. The ACS is also called as automated on-site factories. They are categorised based on the location of the factory and the direction of construction work progress [7].

The sky factory is the most widely implemented configuration among all construction systems. The buildings are constructed from bottom to top, and the sky factory is shifted upwards as the construction progresses. The sky factory can be supported either on the lower floor [10] or its supports [9] or pulled up along the core of the building [11]. Other categories of the bottom to top construction involve centralised or decentralised sky factory which combines the conventional construction methods[7].

The direction of construction and orientation of building in the ground factory can be either horizontal or vertical. Both vertically oriented and horizontally oriented buildings can be constructed using ground factory building push up method [8]. Unlike the sky factory, lifting capacity of the ground factory system determines the maximum height of the building that can be 
constructed [7]. The self-supported ground factory system can also be used for the construction of horizontally oriented buildings.

Off-site and on-site combined factory system has the central operation units utterly independent of the structure being constructed [7]. Both factories are on a fixed location. It is similar to prefabricated construction in which components fabricated from the off-site factory is assembled at the site. Seamless coordination between both factories is essential for efficient automated construction.

The automated construction methods should have a robust monitoring system to ensure safety and quality of construction $[5,6]$. The monitoring systems associated with the existing automated construction method are mainly focusing on task completion status at the subsystem level. Various types of information from sources such as RFID, cameras, sensors and barcodes are used for monitoring purpose in ACSs [8,10-12]. Integrating this information from various operation subsystems are essential to determine the state of the overall constructed building. Crucial management decisions and the overall cost of the construction can highly depend on the right information regarding the ongoing construction. The automated construction, which involves far more complicated equipment and methods compared to the conventional construction method, demands a rigorous monitoring system.

\section{Automated top down construction}

The conventional method of construction progresses from bottom to top, starting from the foundation level. The major cost in an automated construction is associated with lifting. The bottom to top method of automated construction involves lifting of the entire central operation unit after the completion of each floor. For the construction of the low-rise building where reducing the cost is one of the prime criteria, automated top-down construction is the best solution. In automated top-down construction, all activities are carried out at the ground level. This will permit a high level of automation since all the equipment can be installed at the ground level.

The direction of construction progress for the automated top-down construction method described in this paper is from top to bottom [4-6]. Starting from the construction of the topmost floor, the lower floor of the structure is added below the already constructed floor while the latter is lifted in sequence. The load-bearing parts of the structure are modularised into multiple components and assembled during various stages of construction. The 'ground factory and building push-up' method, discussed in the previous section is a similar automated construction method which uses heavy machinery for the construction of an entire floor of a structure at a time $[7,8]$. The modularisation of structural components enables the use of light equipment in the automated topdown construction method. This saves the time and cost of installation and transportation of equipment at the beginning of construction. The compact equipment used in this method can be easily installed and dismantled at the ground level itself. It can also be transported using a small vehicle. Since the machinery is arranged inside the core of the structure to be constructed, it occupies limited space in the construction site. This is a beneficial attribute for construction in space-constrained areas such as cities.

The specific operations and its sequence in the automated top-down construction vary with the configuration of the structure and ACS used. The first category of automated top-down construction uses a specific configuration of the structure which maintains stability even without one support. This category follows operation sequences which allow the connection of one column module at a time. One cycle of operations in automated top-down construction category I can be summarised as follows:

a. Assembly of the topmost beam and column modules of the structure around the ACS supported at each column positions

b. Coordinated lifting of the assembled structure by lifting all supporting platforms simultaneously to one column module height

c. Lower the supporting platform to add column module

d. Connect the column module to the previously installed column module

e. Lift the supporting platform until the load of the structure is transferred completely

f. Repeat steps ' $c$ ' to 'e' for other supporting platforms in the same level of construction

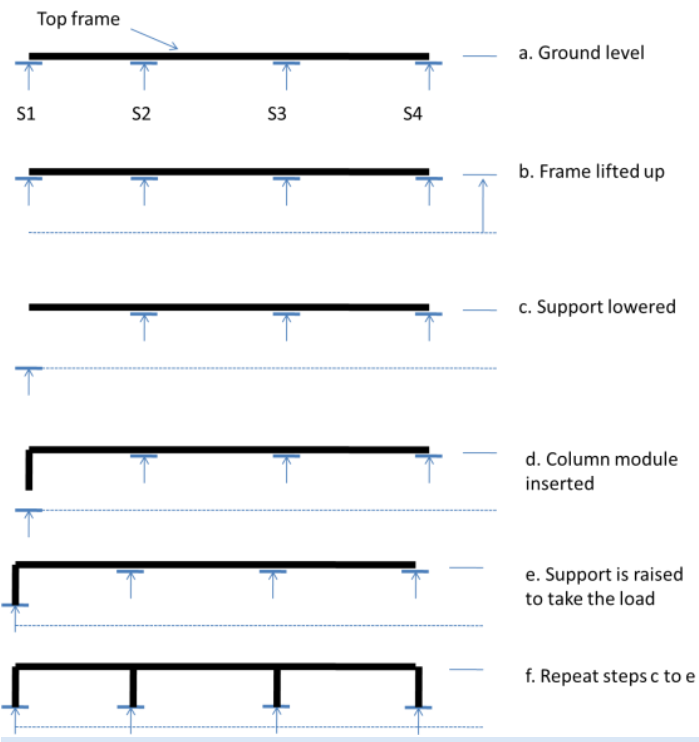

Figure 1. One cycle of operations in automated topdown construction category I 
Figure 1 is a simplified schematic representation of the above steps where S1, S2, .., S4 represents support 1, support 2, ..., support 4. Each cycle of operations completes one level of the structural frame. Several cycles of operations are required to complete a floor of the structural frame. The number of cycles depends on the height of one column module and clear height between two floors of the structural frame.

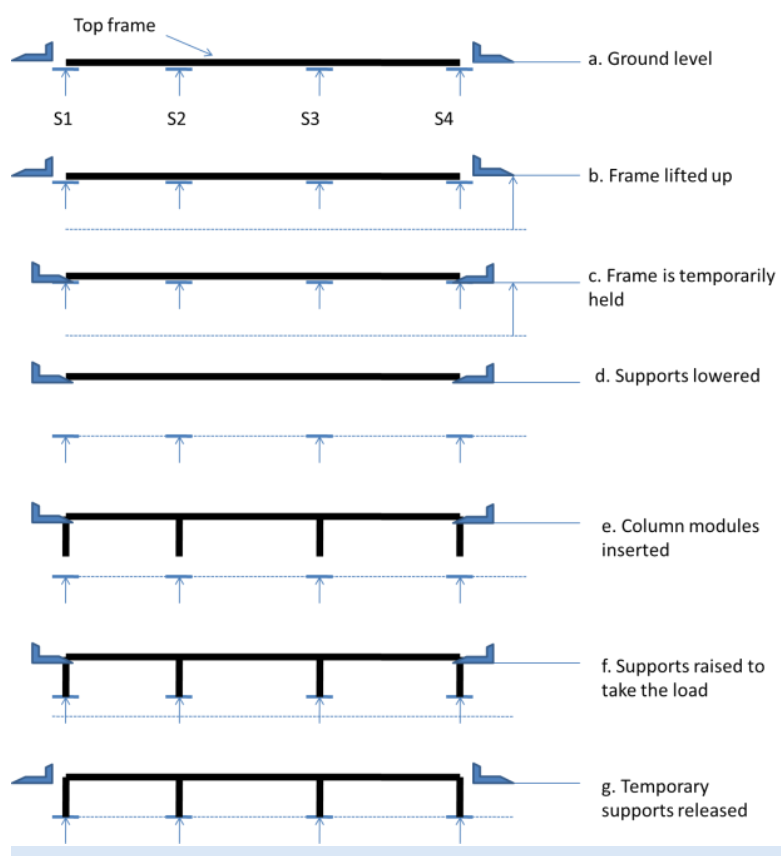

Figure 2. One cycle of operations in automated topdown construction category II

The second category of automated top-down construction uses construction systems which have an additional feature to hold the structure temporarily while the modules of the lower floor are being connected. In this category, the operations will be similar to the previous one except in the case of connections. Instead of connecting one column module at a time, all the column modules in a particular construction level can be connected simultaneously. The construction time reduces considerably compared to the first category. However, the second category demands slightly more intricate equipment which can hold the entire structure at a time. Figure 2 shows a simplified schematic representation of automated top-down construction category II where S1, S2, .., S4 represents support 1, support 2, ..., support 4. This figure is for illustration of the construction concept only.

Automated top-down construction is a highly productive and sustainable method. This involves the construction of the core structural members, segment by segment in a systematic way. The machines required for the construction are placed on the ground, and the structural frame of the building will be pushed up without having the assembly system to climb up with the structure. The advantage of this type of construction is that all the activities are performed at the ground level, and heavy equipment such as tower cranes are not needed.

\section{Methodology}

The main objective of this study is to develop an economical ACS for low rise buildings. The ACS in this project has been developed incrementally. Small prototypes have been developed and tested in the laboratory. The system is evaluated from the functional point of view as well as the cost and efficiency of operations.

Several versions of prototypes were tested with different types of structural and mechanical elements and varying levels of automation. The initial prototype used rectangular timber modules which were connected manually (wooden structure v1) [4]. Only coordinated lifting was automated. This prototype (ACS prototype 1) established the feasibility of the construction scheme. Later, another prototype was implemented to test the automated connection of steel modules using bolts. This prototype used a camera and AI-based image recognition to locate the bolt holes. A custom gripping-alignment system was also implemented to insert the bolts and make the connections. However, the scheme was only partially successful because of the low precision of the fabrication work. The holes on the connecting plates and steel sections had to be perfectly aligned in order to insert the bolts correctly. Even small imperfections in the alignment would cause high friction between the surface of the bolt and the edge of the hole. High precision fabrication considerably increased the cost of construction. Hence, this system was not pursued further. A highly automated construction system (ACS prototype 2) is developed for the construction of a structural system having rectangular steel modules (steel structure v1). Due to the high cost of the prototype and heavy modules of the structural system, further studies were focused on reducing the cost of the prototype and the weight of the structural modules. Later versions used steel pipe sections manually connected using couplers (steel structure v2) [5], [6]. These were found to be economical as well as efficient in operations. The modified construction system (ACS prototype 3 ) is partially automated but has a considerably lower cost compared to the previous prototype. Hydraulic motors (hydraulic motor system v1) and electric motors (electric motor system v1 and v2) were tested for lifting systems. Various prototypes that have been tested are described in this section.

\section{Design of modular structure}

The significant cost associated with automated construction is for lifting operations. Since the central operation unit of automated top-down construction is on 
the ground floor, the only cost of lifting is that of the structure. The modularisation of structural components not only reduce the weight and cost of lifting but also made the components easier to construct.

The first version of the structural system is made of wooden modules of a rectangular cross-section (wooden structure v1). Each module was 400 mm x 200 mm x 400 $\mathrm{mm}$ made of $20 \mathrm{~mm}$ thick wooden planks. Wooden interlocking components connected the modules. This first version of the structural system is used in the first ACS prototype in which connections were made manually by screwing the components (Figures 3 and 4). Similar structural components are used for the second prototype of the ACS (Figure 5). However, the components with the same dimensions are fabricated in steel (steel structure v1). The modules were modified at the top and bottom edge for interlocking, similar to Lego blocks. Additional holes were made at these edges for inserting interlocking pins to secure connection.

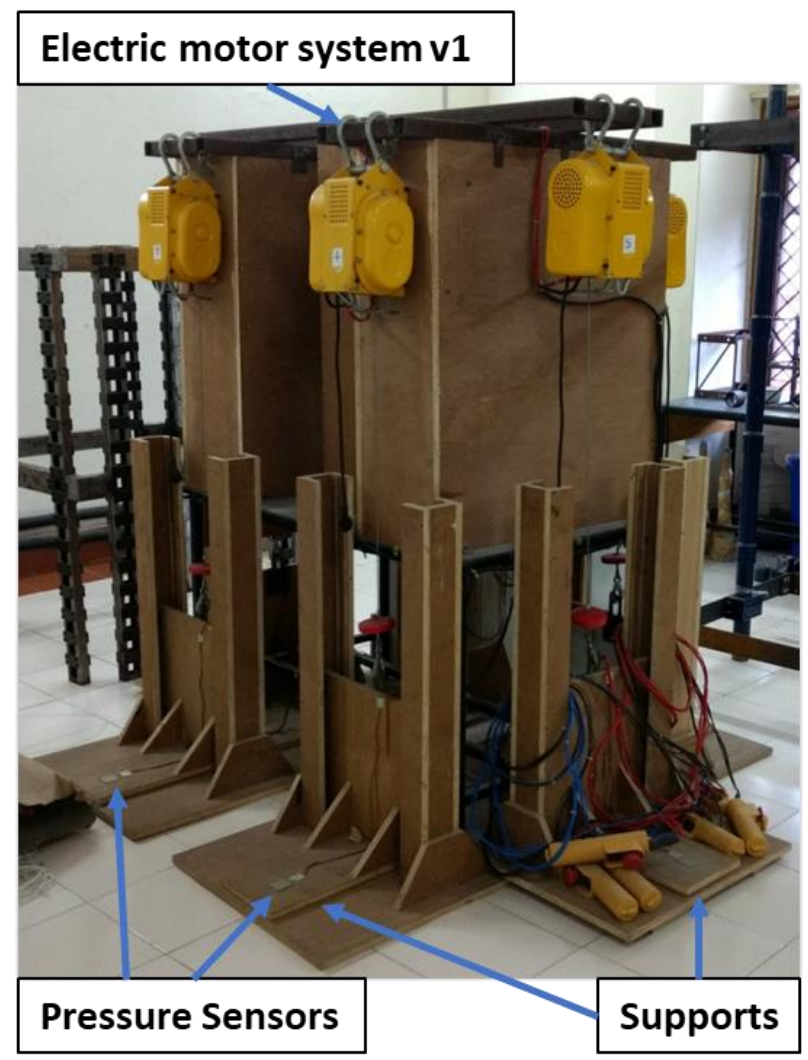

Figure 3. ACS prototype 1

The second version of the structural system is made of mild steel pipe sections with external threading on both ends (steel structure v2). Couplers connect the column modules with internal threading. The connections at the corners of the structure and between bream and column modules are made by custom made universal steel joints. This structural system is used in ACS prototype 3 (Figure
6). Currently, the connections of this structural system are made manually. However, there is a high potential for automating this connection by slight modification in the construction system. An additional facility which includes a gripper to hold the top module while another gripper to hold and rotate the bottom module will help in automating the connection of the structural modules. Further modification of ACS prototype 3 for automating the connections is undergoing.

The structural configuration influences the category of automated top-down construction and the configuration of the construction system. Automated top-down construction category I demand structure with additional columns which ensure stability even without one support (ACS prototype 1 and 3). Automated top-down construction category II can be adopted with the structure having a typical configuration or with additional supports (ACS prototype 2).

\section{Design of automated construction system (ACS)}

The ACS is designed based on the structural configuration and category of automated top-down construction. The construction system has as many supports or lifting platforms as the number of columns in the structure. Automated top-down construction category I require machines capable of lifting and lowering individual support of the structure. Automated top-down construction category II can be implemented only if the construction system can hold the complete structure at any stage of construction. The high weight of lifting, in this case, demands hydraulic systems. That might increase the cost of construction. Electric motors serve economic lifting options. However, the speed and weight of lifting will be reduced. All construction systems in this are partially automated with varying degree of automation. The evolution of the automated top-down construction method through various prototypes are described in the next section.

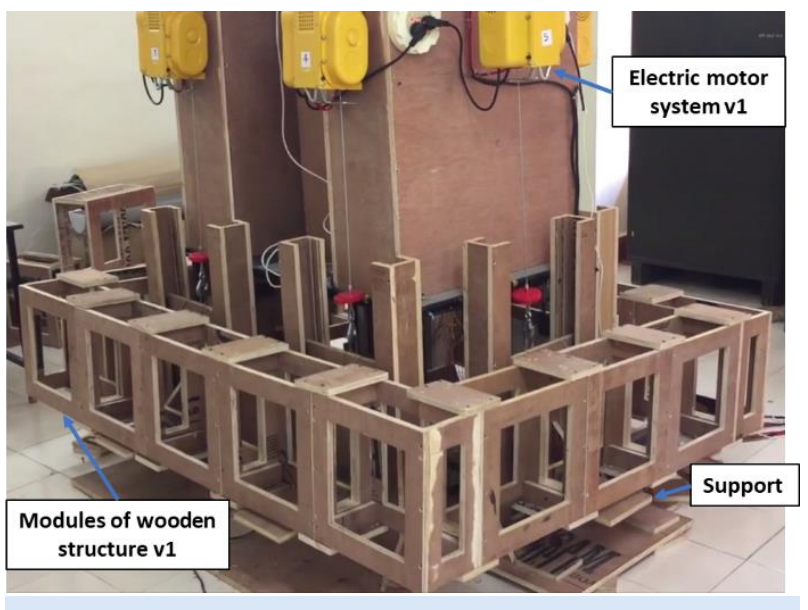

Figure 4. Coordinated lifting of beam assembly at the topmost level using ACS prototype 1 


\section{Development of automated construction system (ACS)}

\section{ACS prototype 1: Wooden structure v1 and electric} motor system $v 1$

ACS Prototype 1 was meant to demonstrate the top-down construction method [4]. The design focus for the construction system was to arrange the machines within the structural frame and to enable coordinated lifting. This prototype follows automated top-down construction category I. The construction system has six lifting machines which can be operated independently or simultaneously based on requirements (Figures 3 and 4). The lifting machines are operated by electric motor hoist with wire ropes (electric motor system v1). The connections in the light wooden structural system (wooden structure v1) were made manually. The data from height sensors and pressure sensors were used for operation and control of the construction system by Arduino microcontrollers. The operations in one cycle of ACS prototype 1 are given in Table 1.

Table 1. Operations in one cycle of ACS prototype 1 and 3

\begin{tabular}{|c|l|}
\hline $\begin{array}{l}\text { Operation } \\
\text { number }\end{array}$ & Operation description \\
\hline 1 & Coordinated lifting \\
\hline 2 & Lowering support no. 1 \\
\hline 3 & Connection of column module step 1 \\
\hline 4 & Lifting support no. 1 \\
\hline 5 & Lowering support no. 2 \\
\hline 6 & Connection of column module step 2 \\
\hline 7 & Lifting support no. 2 \\
\hline 8 & Lowering support no. 3 \\
\hline 9 & Connection of column module step 3 \\
\hline 10 & Lifting support no. 3 \\
\hline 11 & Lowering support no. 4 \\
\hline 12 & Connection of column module step 4 \\
\hline 13 & Lifting support no. 4 \\
\hline 14 & Lowering support no. 5 \\
\hline 15 & Connection of column module step 5 \\
\hline 16 & Lifting support no. 5 \\
\hline 17 & Lowering support no. 6 \\
\hline 18 & Connection of column module step 6 \\
\hline 19 & Lifting support no. 6 \\
\hline & \\
\hline
\end{tabular}

ACS prototype 2: Steel structure v1 and hydraulic motor system $v 1$

The ACS prototype 2 has a custom-designed construction system which consists of hydraulic motors (hydraulic motor system v1) for lifting and connection of steel structural frame (Figure 5). Each machine in this construction system can support the previously constructed structure and perform the construction of two columns simultaneously. The machine has a lifting capacity of 2 ton per support.

The system lifts the partially constructed structure by means of a hydraulic ram controlled by pumps and valves. The pump will be turned off when the signal from the control system prompts the electric motor. This happens when the piston of the hydraulic ram arrives at the required height. The load of the structure will be held by the piston when the valve is closed.

The machine operates with structural modules made of rectangular box sections for beams and columns (steel structure v1). The box sections inter-lock with each other and have holes for connecting modules using interlocking pins. The interlocking pins are inserted automatically by the hydraulic system, at the appropriate time. Besides, there are also load holding pins for temporarily holding the structure before support is lowered. Proximity sensors are installed to ensure proper insertion of pins in each slot. There are light indicators to show the status of load holding pins (when they finish holding or unholding the modules). The supporting platforms are designed to fit the rectangular modules. They also allow the movements of pistons to insert both load-holding pins and interlocking pins from the rear end. The supports have a provision to insert the alignment rod which keeps the module in position and avoid the module from falling off at any situation during lifting.

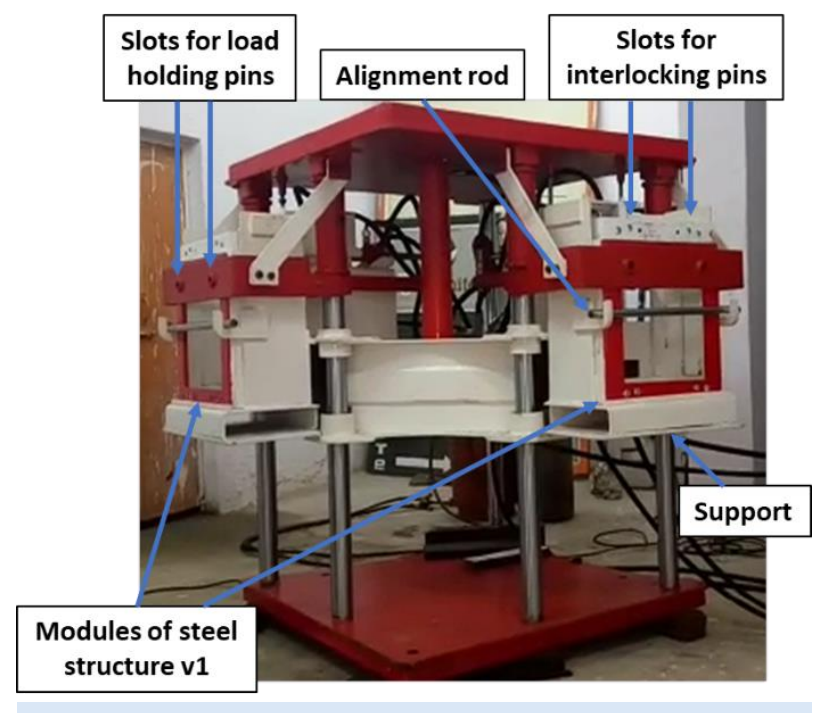

Figure 5. Holding of corner column modules using one machine in ACS prototype 2

The sequence of operation at a support is as follows: In the first cycle, each module is placed on each of the supporting platforms, aligned at the designated place using alignment rod. In the next step, the module is lifted 
by the load lifting cylinder by $50 \mathrm{~mm}$ to interlock with the module above. The interlocking pins are pushed into position by the pin locking cylinder and the load holding pins are retracted. Then the load lifting cylinder lifts the module by one module height. The load holding pins lock into the module and holds it in place. Then the first alignment rod is removed. In the reverse cycle, the load lifting cylinder is lowered by a height of one module height $+50 \mathrm{~mm}$ clearance. The pin locking cylinder is retracted, and the new module is placed. This process repeats until the entire frame is constructed. Note that the operations at just one support are described here. The same operations were carried out simultaneously for all other supports. The ACS prototype 2 follows automated top-down construction category II. The whole operations per cycle for ACS prototype 2 is given in Table 2. This ACS prototype is designed for a structural system with two corner columns. Hence, there are eight columns for the structure and four machines are required to complete the construction of the structural frame (steel structure v1).

Table 2. Operations in one cycle of ACS prototype 2

\begin{tabular}{|c|l|}
\hline $\begin{array}{l}\text { Operation } \\
\text { number }\end{array}$ & Operation description \\
\hline 1 & Coordinated lifting \\
\hline 2 & Holding column modules (8 no.) \\
\hline 3 & Coordinated lowering \\
\hline 4 & Loading column module 1 \\
\hline 5 & Loading column module 2 \\
\hline 6 & Loading column module 3 \\
\hline 7 & Loading column module 4 \\
\hline 8 & Loading column module 5 \\
\hline 9 & Loading column module 6 \\
\hline 10 & Loading column module 7 \\
\hline 11 & Loading column module 8 \\
\hline 12 & Coordinated lifting until interlocking \\
\hline 13 & Connection of column modules (8 no.) \\
\hline 14 & Unholding column modules (8 no.) \\
\hline
\end{tabular}

Even though the construction system was highly efficient in terms of speed and ease of construction, the cost was high. The rectangular modules were heavy $(30.8 \mathrm{~kg})$ and two labours were required to load them into the construction system. This version of the construction system encouraged to look onto lighter structural configuration and economical lifting options.

\section{ACS prototype 3: Steel structure v2 and electric motor system $v 2$}

The ACS Prototype 3 is an improved version of ACS prototype 1 and follows the automated top-down construction category I. The construction system consists of 6 lifting machines with a stepper motor for precise operation (Figure 6). Each machine has a lifting capacity of 2 ton. Similar to ACS prototype 1, the lifting machines can be operated individually and simultaneously. The construction system is controlled by programmable Arduino microcontrollers. The operations and its sequence are the same as that of the ACS prototype 1 (Table 1). However, the structural system is made of steel pipe sections. This reduced the overall weight of the components considerably compared to that of the previous ACS prototype. Therefore, the equipment in this construction system is lighter and compact than the previous version. Even though the speed of construction is reduced, the current version is much more economical.

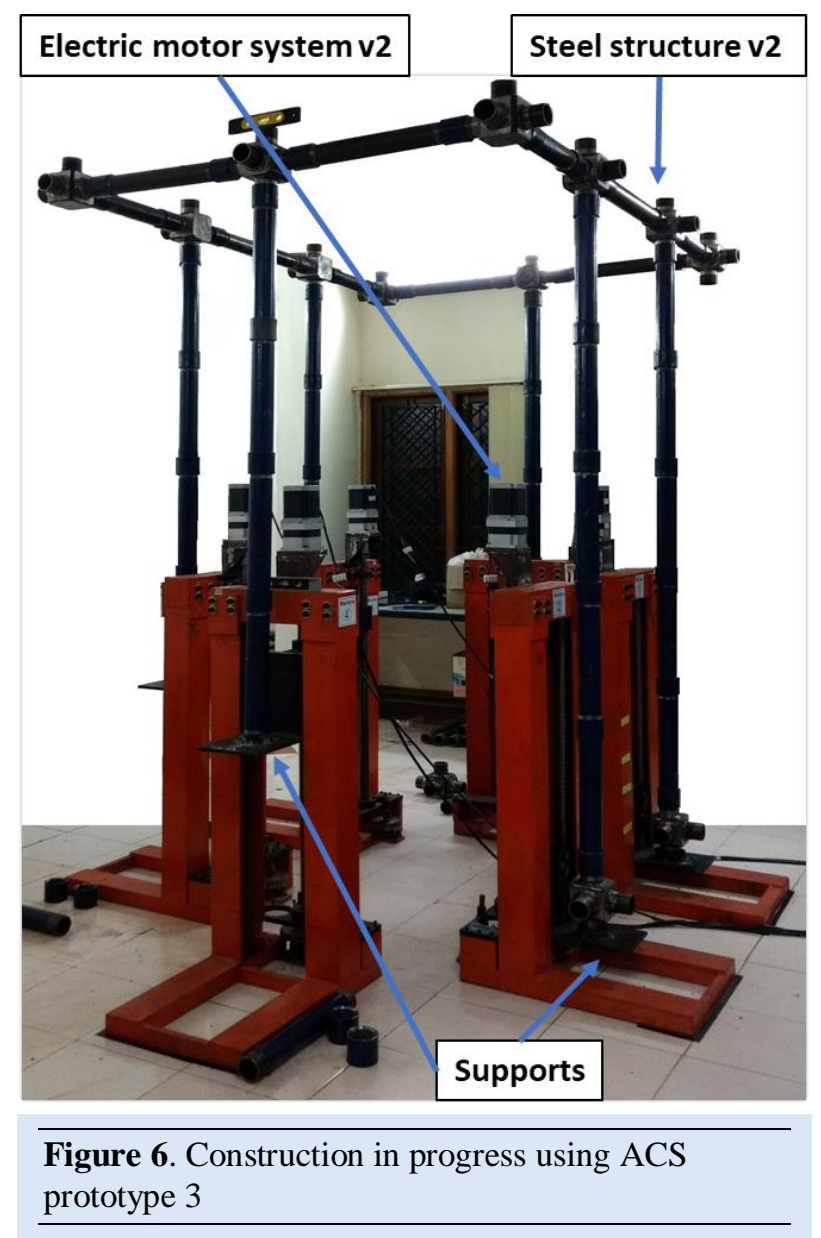

\section{Automated Construction Monitoring}

Monitoring of automated construction is essential to avoid severe construction accidents [5], [6]. Machine learning techniques are widely implemented for construction monitoring from sensor data [13-15]. This paper presents a preliminary framework based on machine learning for the identification of automated construction operations (Figure 7). The sensor measurements collected from the structure during automated top-down construction are supplied as the input data. This data is collected during controlled 
experiments conducted with ACS prototypes in the laboratory condition. The pattern of the signal corresponding to each operation is identified by machine learning techniques.

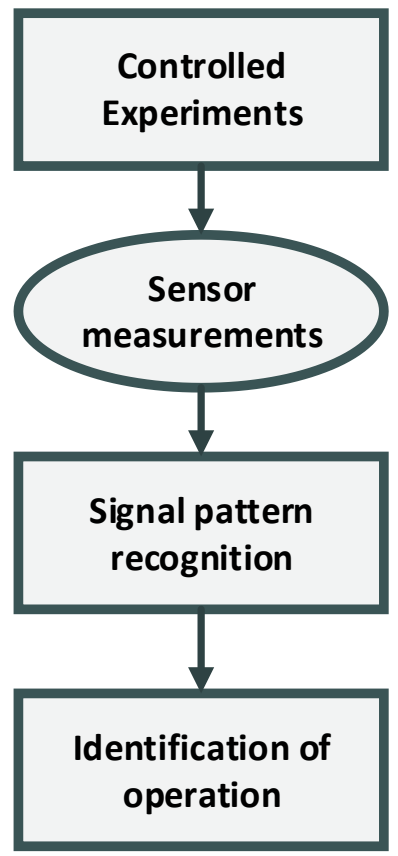

Figure 7. Framework for identification of automated construction operations

The results of the controlled experiments conducted on ACS prototype 3 is analysed in this study. Table 3 shows five major operation classes or states which are experimentally evaluated for classification analysis. The structural system is installed with accelerometers and strain gauges at the location, as shown in Figure 8. The sensor measurements are recorded during experiments. These measurements are used for signal pattern recognition for identifying automated construction operations by supervised learning techniques. Out of six sets of experimental data, five sets were allocated for training the machine learning model, and one set is allotted for testing. The performance of the classification model is determined as a percentage of accurate predictions. A record of actual operations with timestamp is maintained for validation purpose.
Table 3. Major classes of automated construction operations or states which are experimentally evaluated

\begin{tabular}{|l|l|l|}
\hline $\begin{array}{l}\text { S1. } \\
\text { No. }\end{array}$ & $\begin{array}{l}\text { Classes of automated construction } \\
\text { operations }\end{array}$ & $\begin{array}{l}\text { Number of } \\
\text { operations } \\
\text { per class }\end{array}$ \\
\hline 1 & Idle state (Ambient Vibration) & 2 \\
\hline 2 & Coordinated lifting & 3 \\
\hline 3 & Lowering support & 12 \\
\hline 4 & Connection of column module & 12 \\
\hline 5 & Lifting support & 12 \\
\hline \multicolumn{2}{|l|}{ Total number of operations } & 41 \\
\hline
\end{tabular}
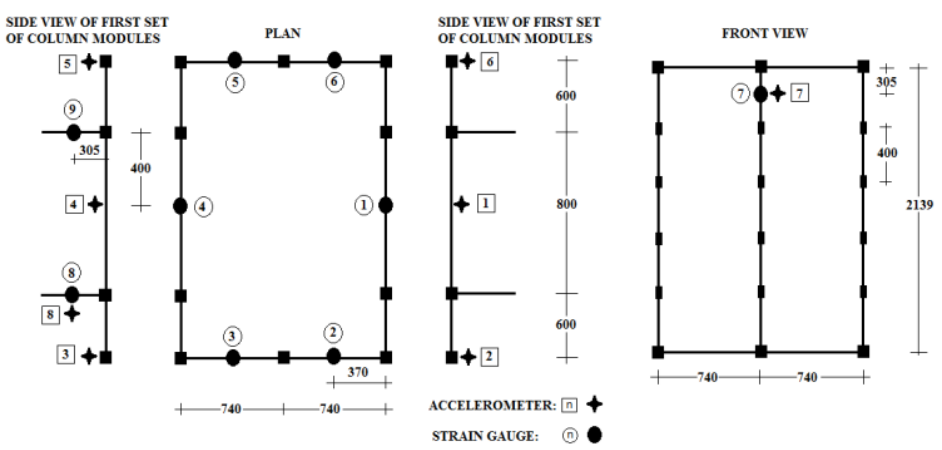

Figure 8. Location of sensors on a structural system constructed by ACS prototype 3 (All dimensions are in $\mathrm{mm}$ )

Support Vector Machines (SVM) is a machine learning technique extensively used for signal pattern recognition and operation identification[16-19]. The current study adopts the SVM classification for identifying automated construction operations. Table 4 shows the classification methods and parameters used for classification analysis. SVM technique involves identification of operation corresponds to each data point through binary classification. During training, the classification model is provided with data points and corresponding labels. The labels of all data points will be negative, except the ones correspond to operation to be identified. For those data points, the labels will be positive. In supervised learning, a hyperplane which separates the data points into classes is identified.

Table 4. SVM classification methods and parameters used for data analysis

\begin{tabular}{|l|l|l|l|}
\hline Sl.No. & Method of classification & Parameters and range of values & $\begin{array}{l}\text { Number } \\
\text { analysis } \\
\text { operation }\end{array}$ \\
\hline 1 & Linear Classification & 10 \\
\hline 2 & $\begin{array}{l}\text { Nonlinear Classification with } \\
\text { Radial Basis Function (RBF) }\end{array}$ & $\begin{array}{l}\text { Error penalty (C): } 10-100 \\
\text { Width of Gaussian kernel }(\sigma): 0.5-1.5\end{array}$ & 110 \\
\hline
\end{tabular}


Table 5. Specifications of ACS prototypes

\begin{tabular}{|l|l|l|l|l|l|l|l|}
\hline \multirow{2}{*}{$\begin{array}{l}\text { ACS } \\
\text { Prototype }\end{array}$} & $\begin{array}{l}\text { Automated } \\
\text { top-down } \\
\text { construction }\end{array}$ & \multicolumn{2}{|l|}{ Structural system } & \multicolumn{2}{l|}{ Construction system } & $\begin{array}{l}\text { Number } \\
\text { of } \\
\text { operations } \\
\text { per cycle }\end{array}$ & $\begin{array}{l}\text { Number of } \\
\text { machines } \\
\text { required for } \\
\text { ACS } \\
\text { prototype }\end{array}$ \\
\cline { 3 - 9 } & & Material & Version & Operation Unit & Version & & \\
\hline 1 & Category I & Wood & 1 & Electric motor & 1 & 19 & 6 \\
\hline 3 & Category II & Steel & 1 & Hydraulic motor & 1 & 14 & 4 \\
\hline
\end{tabular}

The hyperplane is defined by Support Vector Machines. These are data points belong to each operation classes from which maximum distance has to be maintained by the hyperplane. The solution for hyperplane involves parameters whose values can be tuned to obtain a better classification. The current study uses two parameters. The first one is the error penalty $(C)$. The higher value of $C$ increases the penalty for misclassification during training. The second parameter is the width of the Gaussian kernel $(\sigma)$ for the Radial Basis Function classifier.

The patterns in data are best identified when it is represented in terms of features which have the information to discriminate it [20]. The features extracted from moving time windows over the raw data is used for supervised learning. The statistical features extracted from the raw data are maximum, minimum, standard deviation $(\sigma)$, mean and histogram (range: $-3 \sigma$ to $+3 \sigma$ ).

\section{Results and discussion}

Performance of automated construction systems

The prototypes of automated top-down constructed systems are evaluated based on construction time, cost, level of automation, the skill level required for construction and ease of transportation, assembling and disassembling of construction systems. Table 5 shows a brief description of the specifications of each ACS prototype. Table 6 presents a comparison of average cycle time estimated through experimental studies and other details. The experimental studies on all ACS prototypes involve 2 unskilled labour for construction and one labour trained for the operation of the machine. Here, cycle time refers to the time for completing one cycle of operations of a particular construction system. That is time for completion of one level of construction. One floor of the structural system contains several construction levels.

The first ACS prototype is expected to have a longer cycle time compared to others.

Table 6. Comparison of ACS prototypes

\begin{tabular}{|l|l|l|l|l|l|l|}
\hline $\begin{array}{l}\text { ACS } \\
\text { Prototype }\end{array}$ & $\begin{array}{l}\text { Average } \\
\text { cycle time } \\
\text { (minutes) }\end{array}$ & Level of Automation & $\begin{array}{l}\text { Weight of one } \\
\text { machine in } \\
\text { ACS } \\
\text { prototype }(\mathrm{kg})\end{array}$ & $\begin{array}{l}\text { Total weight } \\
\text { of ACS } \\
\text { prototype } \\
\text { (kg) }\end{array}$ & $\begin{array}{l}\text { Cost of one } \\
\text { machine in } \\
\text { ACS } \\
\text { prototype } \\
\text { (INR) }\end{array}$ & $\begin{array}{l}\text { Total cost } \\
\text { of ACS } \\
\text { prototype } \\
\text { (INR) }\end{array}$ \\
\hline 1 & 60 & $\begin{array}{l}\text { Lifting: Automated } \\
\text { Connections: Manual }\end{array}$ & 16 & 100 & 31,666 & 190,000 \\
\hline 3 & 2.65 & $\begin{array}{l}\text { Lifting: Automated } \\
\text { Connection of beam } \\
\text { modules: Manual } \\
\text { Connection of column } \\
\text { modules: Automated }\end{array}$ & 500 & 2000 & 600,000 & $2,400,000$ \\
\hline & 16 & $\begin{array}{l}\text { Lifting: Automated } \\
\text { Connections: Manual }\end{array}$ & 40 & 250 & 200,000 & $1,200,000$ \\
\hline
\end{tabular}


The main reason for high cycle time is the manual process of alignment and connections. This problem can be solved either by introducing advanced robotic technologies for alignment and connections. The level of automation to be adopted at each stage of construction should be based on the trade-off between time and cost of construction. The second ACS prototype which follows automated topdown construction category II has least cycle time. There are two main reasons for the best cycle time. All modules of the column are connected simultaneously, and the hydraulic motor is faster compared to the electric motor. However, the second ACS prototype is too costly for a low-rise building construction. Even though the ACS prototype 1 is the least expensive among all prototypes, it is far too preliminary for actual construction sites. That makes ACS prototype 3 a better option for an affordable construction method with reasonably good cycle time.

Comparing the ease of construction, ACS prototype 2 is the best option with the highest level of automation. The few human involvements in the construction are the loading of column modules and connection of beam modules at the beginning of construction. These activities do not require a high skill. This ACS prototype has an option to manually operate the construction system along with fully automated operation cycle. The third ACS prototype involves the manual connection of column modules. This operation can also be performed by unskilled labour. The connections are relatively easier compared to that of ACS prototype 1 and any conventional methods. The connection of timber modules in ACS prototype 1 by screwing can be easily made by unskilled labour. However, maintaining the level of the components requires some skill level.

The ACS prototype 3 is lighter than ACS prototype 2 and most compact among all while comparing individual machines. Even though ACS prototype 1 is lightest among all prototypes, it is bulky. Transportation of this prototype is simple but the initial set up, assembling and disassembling requires skilled labour. Transportation, assembling and disassembling will be easy for ACS prototype 3, compared to ACS prototype 2. This is because all of the 6 machines in ACS prototype 3 are light and compact. Each of these machines can be moved using a simple metal trolley. The setting up and dismantling at construction sites do not require any skilled labour. The individual machines in the second ACS prototype are heavy, large and contain numerous sensors. It requires a skilled forklift operator for shifting and placing it without damaging the sensors. The initial set up after placing the individual machines and dismantling also requires a welltrained operator.

Comparing the overall performances in each criterion, ACS prototype 3 is the best economical option for the construction of low-rise buildings. While construction time is the governing criteria, ACS prototype 2 is the best option. In either case, further modification has to be made in the ACS prototypes to secure the sensors and for better performance, before implementing them in the actual construction site.

Table 7. Operation wise best prediction results

\begin{tabular}{|l|l|l|l|l|l|}
\hline Sl.No. & Operation/State & Classification type & C & $\sigma$ & $\begin{array}{l}\text { Best percentage of } \\
\text { correct predictions }\end{array}$ \\
\hline 1 & Coordinated lifting & Nonlinear Classification & 10 & 0.9 & $96 \%$ \\
\hline 2 & Coordinated lifting & Nonlinear Classification & 20 & 0.9 & $96 \%$ \\
\hline 3 & Coordinated lifting & Nonlinear Classification & 30 & 0.9 & $96 \%$ \\
\hline 4 & Coordinated lifting & Nonlinear Classification & 40 & 0.9 & $96 \%$ \\
\hline 5 & Coordinated lifting & Nonlinear Classification & 50 & 0.9 & $96 \%$ \\
\hline 6 & Coordinated lifting & Nonlinear Classification & 60 & 0.9 & $96 \%$ \\
\hline 7 & Coordinated lifting & Nonlinear Classification & 70 & 0.9 & $96 \%$ \\
\hline 8 & Coordinated lifting & Nonlinear Classification & 80 & 0.9 & $96 \%$ \\
\hline 9 & Coordinated lifting & Nonlinear Classification & 90 & 0.9 & $96 \%$ \\
\hline 10 & Coordinated lifting & Nonlinear Classification & 100 & 0.9 & $96 \%$ \\
\hline 11 & Connection of column module & Linear Classification & 10 & & $90 \%$ \\
\hline 12 & Lowering support & Linear Classification & 10 & & $86 \%$ \\
\hline 13 & Lowering support & Linear Classification & 20 & & $86 \%$ \\
\hline 14 & Lifting support & Linear Classification & 80 & & $80 \%$ \\
\hline 15 & Lifting support & Linear Classification & 90 & & $80 \%$ \\
\hline 16 & Lifting support & Linear Classification & 100 & & $80 \%$ \\
\hline 17 & Idle & Linear Classification & 60 & & $72 \%$ \\
\hline
\end{tabular}




\section{Identification of automated construction operations}

The strain and accelerometer measurements collected during automated construction by ACS prototype 3 are used for identifying construction operations by SVM classification. The features extracted from raw measurement data is used for training the machine learning model. After the training, the machine learning model is given with unseen data to identify automated construction operations. The prediction results are compared with the digital record of the actual operations to compute the accuracy of prediction of the classification model. 600 models were trained by varying the values of $\mathrm{C}$ and $\sigma$. The optimum values of the parameters are identified from the classification models which has the highest accuracy of prediction.

The operation wise best prediction results and optimum values of parameters are given in Table 7. Coordinated lifting is the best-identified operation. Unlike other operations, this is identified through nonlinear classification. The identification of this operation seems to be unaffected by the error penalty. Clear peaks in acceleration measurements in the beginning and end of the operation characterise this operation. The features extracted from the data represent the patterns in the whole data.

All other operations are recognised through linear classification. However, the optimum value of the error penalty varies in each case. The idle case has the lowest number of data points, which resulted in less accuracy in identification in the initial analysis. The data corresponds to idle class is augmented to avoid unbalanced data points during further training. The data augmentation seems to improve the identification. The operations other than coordinated lifting lack repeating patterns. Operations like lowering of support and idle sometimes have similar patterns. Hence, careful selection of features is essential for better identification.

\section{Conclusions and future work}

This paper presents the incremental development of an automated construction system (ACS) for low-rise buildings through laboratory prototypes. The constructability issues which can be identified only through implementation were addressed in each stage of development. The ACS prototypes were accessed by proposed performance parameters based on functionality and cost. Based on overall performance, ACS prototype 3 is identified as the best economical option for the construction of low-rise buildings. This is a partially automated system where the structural modules are connected manually. When the speed of construction is more important than cost, the ACS prototype 2 which has the highest level of automation is the best solution.
A preliminary framework for automated construction monitoring is also explored in the study. The machine learning-based framework is applied to identify the operations of ACS prototype 3. The strain and acceleration measurements collected during automated construction are used for supervised learning by SVM classifiers. Error penalty $(\mathrm{C})$ and Gaussian kernel width $(\sigma)$ are selected as turning parameters to improve operation identification. Most of the operations are identified reasonably well and the best identification accuracy is $96 \%$. The coordinated lifting operation which is having clearly distinguishable and repetitive acceleration measurements is the best-identified operation.

The laboratory prototypes of automated construction implemented in this project demonstrate the potential for faster construction of structural frames of low-rise buildings. The possibility for automated monitoring of the operations is also demonstrated by the experiments conducted in this work. The prospect of machine learning techniques for identifying automated construction operations from sensing data is evaluated.

Even though the ACS prototypes developed in this study shows promising performance, they have to be modified appropriately before implementing it to construction sites. The sensors have to be protected from dust and possible damages during transportation and installation. Automating the connections of beam and column modules is currently under study. The further development of the automated monitoring system is also undergoing.

\section{Acknowledgements}

The support provided by the faculties and staffs from the Structural Engineering Laboratory, Building Automation Laboratory, and Civil Engineering Workshop at IIT Madras is sincerely acknowledged. The scholarship from the Ministry of Human Resource Development (MHRD), India and Curtin International Postgraduate Research Scholarship (CIPRS) and Research Stipend Scholarship from Curtin University, Australia support the research works of the first author. The funding for this research project is provided by the Department of Science and Technology (DST), India through the grants DST/TSG/AMT/2015/234 and IMP/2018/000224.

\section{References}

[1] Bock, T. (2015). "The future of construction automation: Technological disruption and the upcoming ubiquity of robotics." Automation in Construction, 59, 113-121. https://doi.org/10.1016/j.autcon.2015.07.022

[2] Bureau of Labor Statistics. (2018). National census of fatal occupational injuries in 2017. (Issue December 18). https://www.bls.gov/news.release/pdf/cfoi.pdf 
[3] Cai, S., Ma, Z., Skibniewski, M. J., \& Bao, S. (2019). "Construction automation and robotics for high-rise buildings over the past decades: A comprehensive review." Advanced Engineering Informatics, 42, 100989. https://doi.org/10.1016/J.AEI.2019.100989

[4] Raphael, B., Rao, K. S. C., \& Varghese, K. (2016). "Automation of modular assembly of structural frames for buildings." Proceedings, 33rd International Symposium on Automation and Robotics in Construction (ISARC 2016), Auburn, AL, USA, July 18-21, pp. 412420. https://doi.org/10.22260/ISARC2016/0050

[5] Harichandran, A., Raphael, B., \& Mukherjee, A. (2019a). "Identification of the structural state in automated modular construction." Proceedings, 36th International Symposium on Automation and Robotics in Construction (ISARC 2019), Banff, AB, Canada, May 21-24, $\mathrm{pp}$. 187-193. https://doi.org/10.22260/ISARC2019/0026

[6] Harichandran, A., Raphael, B., \& Mukherjee, A. (2019b). "Determination of automated construction operations from sensor data using machine learning." Proceedings, 4th International Conference on Civil and Building Engineering Informatics, Sendai, Miyagi, Japan, Nov. 7-8, pp. 77-84.

[7] Bock, T., \& Linner, T. (2016). Site Automation Automated/Robotic On-site Factories. Cambridge University Press.

[8] Sekiguchi, T., Honma, K., Mizutani, R., \& Takagi, H. (1997). "The development and application of an automatic building construction system using push-up machines." Proceedings, 14th International Symposium on Automation and Robotics in Construction (ISARC), Pittsburgh, USA, pp. 321-328. https://doi.org/10.22260/isarc1997/0040

[9] Wakisaka, T., Furuya, N., Inoue, Y., \& Shiokawa, T. (2000). "Automated construction system for high-rise reinforced concrete buildings." Automation in Construction, 9(3), 229-250. https://doi.org/10.1016/S0926-5805(99)00039-4

[10] Yamazaki, Y., \& Maeda, J. (1998). "The SMART system: an integrated application of automation and information technology in production process." Computers in Industry, 35(1), 87-99. https://doi.org/10.1016/S0166-3615(97)00086-9

[11] Sakamoto, S., \& Mitsuoka, H. (1994). "Totally Mechanized Construction System for High-Rise Buildings (T-UP System)." Proceedings, 11th International Symposium on Automation and Robotics in Construction (ISARC), Brighton, UK, pp. 465-472.

[12] Miyakawa, H., Ochiai, J., Oohata, K., \& Shiokawa, T. (2000). "Application of automated building construction system for high-rise office building." Proceedings, 17th International Symposium on
Automation and Robotics in Construction (ISARC), Taipei, Taiwan, pp. 1-6. https://doi.org/10.22260/ISARC2000/0083

[13] Akhavian, R., \& Behzadan, A. H. (2014). "Construction activity recognition for simulation input modeling using machine learning classifiers." Proceedings, Winter Simulation Conference 2014, Savannah, GA, USA, Dec. 7-10, pp. 3296-3307. https://doi.org/10.1109/WSC.2014.7020164

[14] Cheng, C.-F., Rashidi, A., Davenport, M. A., \& Anderson, D. V. (2017). "Activity analysis of construction equipment using audio signals and support vector machines." Automation in Construction, 81, 240253. https://doi.org/10.1016/J.AUTCON.2017.06.005

[15] Joshua, L., \& Varghese, K. (2011). “Accelerometerbased activity recognition in construction." Journal of Computing in Civil Engineering, 25(5), 370-379. https://doi.org/10.1061/(ASCE)CP.1943-5487.0000097

[16] Harichandran, A., Raphael, B., \& Varghese, K. (2018). "Inferring construction activities from structural responses using support vector machines." Proceedings, 35th International Symposium on Automation and Robotics in Construction (ISARC 2018), Berlin, Germany, Jul. 25-28, pp. 332-339. https://doi.org/10.22260/ISARC2018/0047

[17] Golparvar-Fard, M., Heydarian, A., \& Niebles, J. C. (2013). "Vision-based action recognition of earthmoving equipment using spatio-temporal features and support vector machine classifiers." Advanced Engineering Informatics, 27(4), 652-663. https://doi.org/10.1016/J.AEI.2013.09.001

[18] Twomey, N., Diethe, T., Fafoutis, X., Elsts, A., McConville, R., Flach, P., \& Craddock, I. (2018). “A comprehensive study of activity recognition using accelerometers." Informatics, 5(2), 1-37. https://doi.org/10.3390/informatics5020027

[19] Burges, C. J. C. (1998). "A tutorial on support vector machines for pattern recognition." Data Mining and Knowledge Discover, 2, 121-167. https://doi.org/10.1023/A:1009715923555

[20] Bishop, C. M. (2006). Pattern Recognition and Machine Learning. (M. Jordan, J. Kleinberg, \& B. Schölkopf (eds.)). Springer. 